\title{
Novel Mutations of the GNE Gene in Distal Myopathy with Rimmed Vacuoles Presenting with Very Slow Progression
}

\author{
Yasuko Ikeda-Sakai ${ }^{\text {a }}$ Yasuhiro Manabe $^{a} \quad$ Daiki Fujii $^{a}$ \\ Syoichiro Kono ${ }^{a}$ Hisashi Narai ${ }^{a}$ Nobuhiko Omori ${ }^{a}$ \\ Ichizo Nishino ${ }^{c}$ Koji Abe ${ }^{b}$ \\ ${ }^{a}$ Department of Neurology, National Hospital Organization Okayama Medical \\ Center, and ${ }^{b}$ Department of Neurology, Graduate School of Medicine, Dentistry \\ and Pharmaceutical Sciences, Okayama University, Okayama, and \\ 'Department of Neuromuscular Research, National Institute of Neuroscience, \\ National Center of Neurology and Psychiatry, Tokyo, Japan
}

\section{Key Words}

Distal myopathy with rimmed vacuoles - UDP-N-acetylglucosamine-2-epimerase and $\mathrm{N}$-acetylmannosamine kinase $\cdot$ Sialic acid

\begin{abstract}
We report novel compound heterozygous mutations of the UDP-N-acetylglucosamine-2epimerase and $\mathrm{N}$-acetylmannosamine kinase (GNE) gene, c.302G $>\mathrm{A}$ (p.R101H) and c.617$4 A>G$, in a Japanese family with distal myopathy with rimmed vacuoles (DMRV) presenting with slow progression. The three patients could stand and walk even 36,34 , and 39 years after onset, respectively, although affected individuals become wheelchair bound on average 12 years after onset of the disease. The clinical spectrum of DMRV seems to be wider than previously thought in terms of both the clinical course and the severity of the disease.
\end{abstract}

\section{Introduction}

Distal myopathy with rimmed vacuoles (DMRV), also called hereditary inclusion body myopathy (hIBM), is an juvenile-to-adult-onset autosomal-recessive disorder clinically characterized by preferential involvement of the lower leg muscles, especially the tibialis anterior muscle [1,2]. Affected individuals become wheelchair bound on average 12 years after onset of the disease [1]. Muscle pathology is characterized by 
rimmed vacuoles, in addition to fiber size variation. The disease is caused by mutations in $G N E$, encoding a protein with activities of UDP-N-acetylglucosamine-2-epimerase and $\mathrm{N}$-acetylmannosamine kinase (GNE), which is an essential enzyme in sialic acid biosynthesis [3-12]. We characterize novel mutations of the GNE gene in a Japanese family with DMRV presenting with slow progression.

\section{Case Reports}

Patient 1

A 64-year-old man had developed an insidious steppage gait at age 28. Within one year, he noticed weakness of the finger flexors/extensors. Muscle weakness and atrophy of the four limbs, which were more dominant in distal than proximal parts, gradually progressed. He became unable to write at age 62. He had a history of diabetes mellitus and liver dysfunction from alcoholism. His elder brother and sister had similar symptoms (fig. 1). He had no history of drinking excessive milk or eating swallow's nests, which are rich in sialic acid. On admission, his general physical examination was normal. On neurological examination, cranial nerves were intact. Motor examination revealed $0-1 / 5$ strength in the distal part of the four limbs with muscle atrophy in bilateral digitorum extensor and flexor muscles, wrist extensor and flexor muscles, toe extensor and flexor muscles, tibialis anterior, and

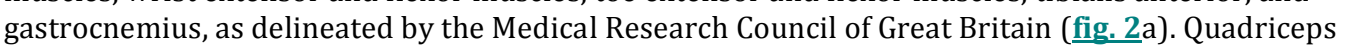
and truncal muscles were spared. He could stand and walk unaided with a steppage gait. Biceps and triceps reflexes were diminished, although ankle and knee jerks were normal. Bilateral Babinski reflexes were absent. Vibration perception was mildly decreased in the distal part of the four limbs. The rest of the neurological examination was unremarkable. Serum creatine kinase (CK) and lactic dehydrogenase (LDH) were normal. Myoglobin was mildly elevated. Anti-Jo-1 antibody was undetected. Computed tomography (CT) scan revealed skeletal muscle atrophy in the extremities and fatty tissue infiltration in bilateral triceps brachii, posterior muscles of the thigh, and muscles of the lower legs. The quadriceps muscles were well preserved (fig. 2b-d). Electromyography of the upper and lower extremities demonstrated myogenic conversion with low amplitude motor unit potential. Muscle biopsy from the left biceps brachii revealed scattered fibers with rimmed vacuoles in addition to marked variation in fiber size (fig. 2e, f).

DNA was extracted from peripheral blood lymphocytes after informed consent was obtained. Each exon and flanking sequences of the GNE gene were amplified by polymerase chain reaction and the amplified fragments were directly sequenced. We identified a novel compound heterozygote of G-to-A transition at nucleotide position c.302 (c.302G>A) in exon 3 , which is predicted to change an amino acid at codon 101 from arginine to histidine (p.R101H), and an A-to-G transition at nucleotide position c.617-4 (c.617-4A>G) in intron 3 (fig. 2g, h).

\section{Patient 2}

The patient was the elder brother of patient 1 (fig. 1). He complained of muscle weakness in both feet at age 36. He also developed muscle weakness in both finger flexors/extensors at age 43. Muscle weakness and atrophy of the four limbs, which were more dominant in distal than proximal parts, gradually progressed. Neurological examination at age 70 demonstrated muscle weakness and atrophy in the distal part of the four limbs and reduced deep tendon reflexes. He could stand and walk unaided with a steppage gait. Serum CK and myoglobin were normal. LDH was mildly elevated. CT scan revealed skeletal muscle atrophy in the extremities and fatty tissue infiltration in bilateral triceps brachii, posterior muscles of the thigh, and muscles of the lower legs. The quadriceps muscles were well preserved (table 1). The same mutations as in patient 1 were detected.

\section{Patient 3}

The patient was the elder sister of patient 1 (fig. 1). She complained of muscle weakness in both feet at age 29. She also developed muscle weakness in both finger flexors/extensors at age 40. Muscle weakness and atrophy of the four limbs, which were more dominant in distal than proximal parts, gradually progressed. Neurological examination at age 68 demonstrated muscle weakness and atrophy in the distal part of the four limbs and reduced deep tendon reflexes. Serum CK was mildly elevated. She could walk with a stick and steppage gait (table 1). The same mutations as in patient 1 were detected. 


\section{Discussion}

We herein report novel compound heterozygous mutations of the GNE gene in a Japanese family with DMRV presenting with slow progression. To date, more than 50 different GNE mutations in DMRV have been reported to cause the disease. GNE, a ratelimiting enzyme that catalyses the initial two steps in the biosynthesis of sialic acid has two functional domains that work independently: the epimerase domain in the amino (N)-terminals, and the kinase domain in the carboxy (C)-terminus. Homozygous M712T is the most common mutation in Middle Eastern Jews, and homozygous V572L among Japanese patients [3-11]. In many other ethnic groups, compound heterozygous mutations have been reported [3-11]; however, the phenotypes are not fully described. The three patients could stand and walk even 36,34 , and 39 years after onset, respectively. Affected individuals become wheelchair bound within an average of 12 years after disease onset [1]. In our three patients, muscle CT revealed the typical distribution of muscle involvement previously reported in DMRV. The tibialis anterior, hamstrings, and adductors were severely affected, but the quadriceps muscles were well preserved. Although it was not clear why the quadriceps muscles of our patients were spared for so many years and there has been no correlation between genotype and phenotype with more than 50 mutations reported, our patients presented with the slowest progression of previously reported patients with DMRV in Japan. Thus, the clinical spectrum of DMRV seems to be wider than previously thought in terms of both the clinical course and the severity of the disease.

Nishino et al. [3] reported a healthy 67-year-old person of Persian Jewish origin carrying the common M712T GNE gene mutation in a homozygous form, suggesting incomplete penetrance of the disease. The results indicate that a modifier gene might be responsible for the phenotype differences, although no such modifier genes have been identified. Our finding of a novel mutant allele in a Japanese patient with DMRV expands on the genetic heterogeneity of this disease and allows for a broader phenotypic-genotypic assessment. The three patients had no history of drinking excessive milk or eating swallow's nests, which are rich in sialic acid. Although it is difficult to explain why this novel mutation leads to unusually slow progression of the disease, further investigation of the correlations among genotypes, enzyme activities and phenotypes is warranted. 
Table 1. Clinical and laboratory findings of distal myopathy with rimmed vacuoles in three Japanese patients

\begin{tabular}{|c|c|c|c|}
\hline & Patient 1 & Patient 2 & Patient 3 \\
\hline Sex & Male & Male & Female \\
\hline Age, years & 64 & 70 & 68 \\
\hline Age at onset, years & 28 & 36 & 29 \\
\hline Initial presentation & Foot drop & Foot drop & Foot drop \\
\hline \multicolumn{4}{|l|}{ Medical Research Council scales } \\
\hline Neck flexor muscles & 4 & 4 & 4 \\
\hline \multicolumn{4}{|l|}{ Upper limbs } \\
\hline Deltoid & 4 & 4 & 5 \\
\hline Biceps & 4 & 4 & 4 \\
\hline Triceps & 3 & 3 & 4 \\
\hline Intrinsic hand muscles & 0 & 1 & 1 \\
\hline \multicolumn{4}{|l|}{ Lower limbs } \\
\hline Quadriceps & 4 & 4 & 4 \\
\hline Hamstrings & 4 & 4 & 4 \\
\hline Tibialis anterior & 0 & 0 & 0 \\
\hline Gastrocnemius & 2 & 1 & 1 \\
\hline Quadriceps sparing & Yes & Yes & Yes \\
\hline Creatine kinase (reference range: $62-287 \mathrm{IU} / \mathrm{l}$ ) & 90 & 184 & 293 \\
\hline \multicolumn{4}{|l|}{ CT imaging fatty infiltration } \\
\hline Quadriceps & - & - & - \\
\hline Hamstrings & ++ & +++ & +++ \\
\hline Tibialis anterior & ++++ & ++++ & ++++ \\
\hline Gastrocnemius & ++++ & ++++ & ++++ \\
\hline Muscle biopsy & $\begin{array}{l}\text { Myopathic changes with } \\
\text { scattered fibers with } \\
\text { rimmed vacuoles }\end{array}$ & not examined & not examined \\
\hline Current status & Walk unaided & Walk unaided & Walk with assistance \\
\hline
\end{tabular}

$+=0-25 \%$ of muscle fibers with fatty infiltration. $++=25-50 \%$ of muscle fibers with fatty infiltration. $+++=50-$ $75 \%$ of muscle fibers with fatty infiltration. $++++=75-100 \%$ of muscle fibers with fatty infiltration.

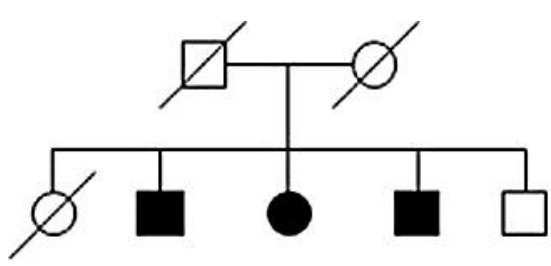

patient 2 patient 3 patient 1

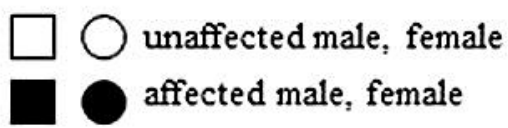

Fig. 1. Pedigree of the patients' family. 

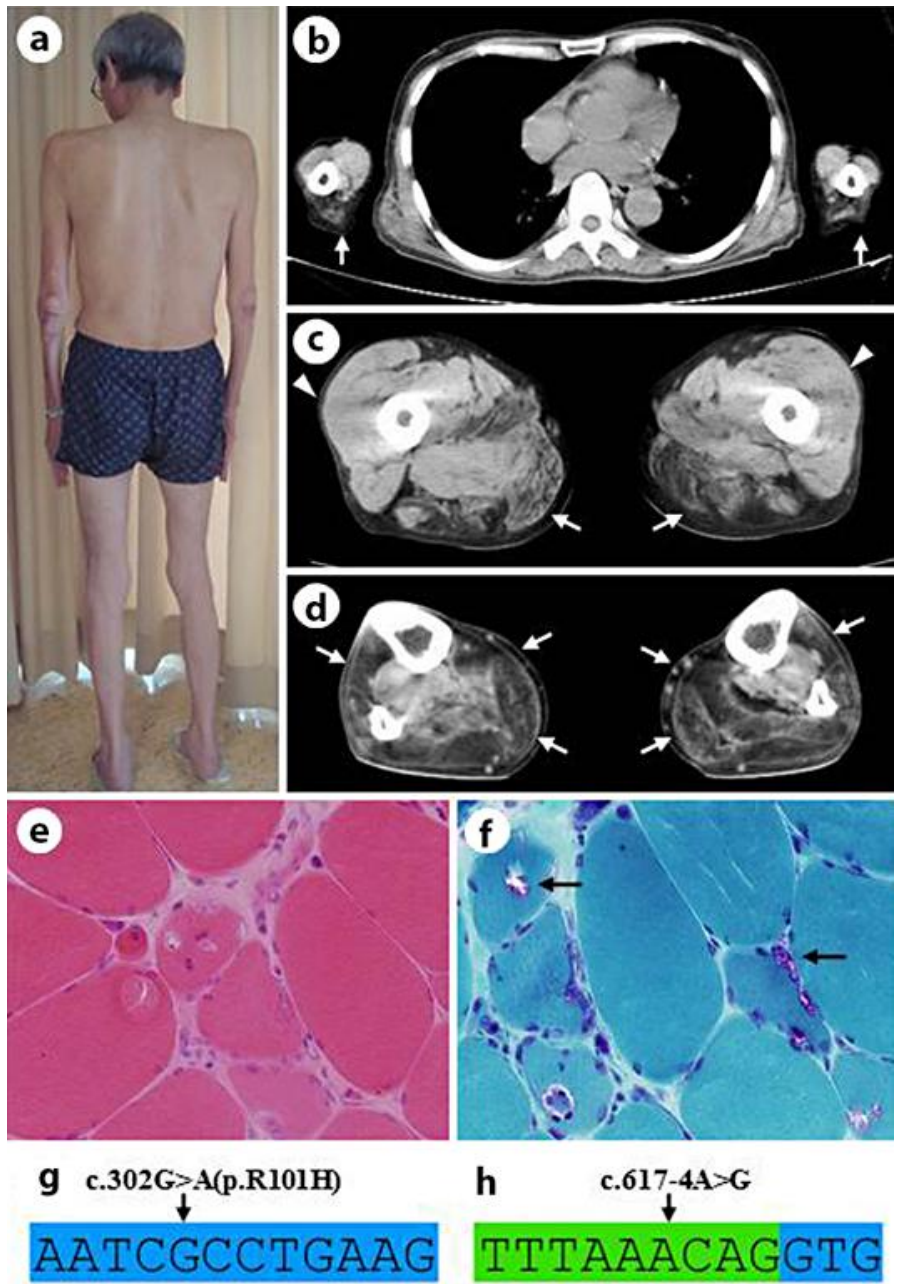

h $\quad$ c.617-4.A $>\mathrm{G}$

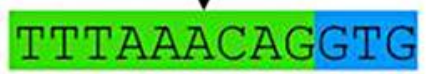

\section{$\begin{array}{llll}N & R & L & K\end{array}$}

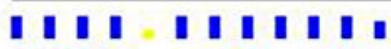
AATCRCCTGAAG
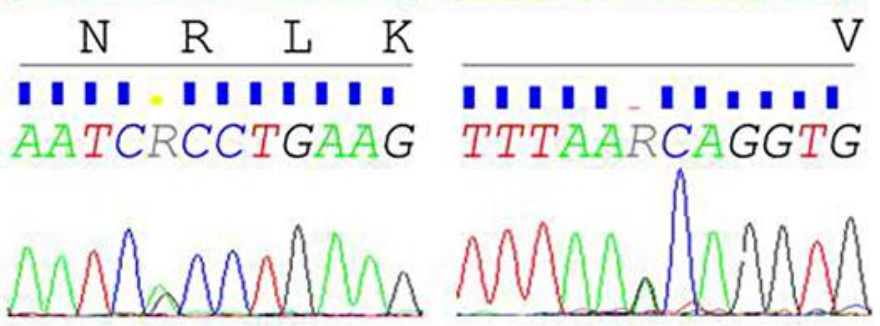

Fig. 2. Image of patient 1 showing muscular atrophy in the distal part of the four limbs (a). CT scan showing skeletal muscle atrophy in the extremities and a low-density area in triceps brachii, posterior muscles of the thigh, and muscles of the distal leg, which showed fatty tissue infiltration of muscles (arrows, b-d). The quadriceps muscles were well preserved (arrowheads, c). In muscle biopsy of the biceps brachii, there is marked variation in fiber size with hypertrophic and small angular fibers, and mild interstitial fibrosis (e) (hematoxylin and eosin), rimmed vacuoles (arrow) are prominent in small fibers $(f)$ (modified Gomori trichrome) $(\times 100)$. Sequence chromatograms of the two heterozygous missense mutations. G-to-A transversion at nucleotide 302 in exon 3 results in a conservative amino acid change $(\mathrm{R} 101 \mathrm{H})(\mathrm{g})$, and A-to-G transition at nucleotide c.617-4 in intron 3 results in a missense mutation (h). 


\section{References}

-1 Nonaka I, Sunohara N, Ishiura S, Satoyoshi E: Familial distal myopathy with rimmed vacuole and lamellar (myeloid) body formation. J Neurol Sci 1981;51:141-155.

-2 Argov Z, Yarom R: 'Rimmed vacuole myopathy' sparing the quadriceps: a unique disorder in Iranian Jews. J Neurol Sci 1984;64:33-43.

-3 Nishino I, Noguchi S, Murayama K, Driss A, Sugie K, Oya Y, Nagata T, Chida K, Takahashi T, Takusa Y, Ohi T, Nishimiya J, Sunohara N, Ciafaloni E, Kawai M, Aoki M, Nonaka I: Distal myopathy with rimmed vacuoles is allelic to hereditary inclusion body myopathy. Neurology 2002;59:1689-1693.

4 Arai A, Tanaka K, Ikeuchi T, Igarashi S, Kobayashi H, Asaka T, Date H, Saito M, Tanaka H, Kawasaki S, Uyama E, Mizusawa H, Fukuhara N, Tsuji S: A novel mutation in the GNE gene and a linkage disequilibrium in Japanese pedigrees. Ann Neurol 2002;52:516-519.

5 Tomimitsu H, Ishikawa K, Shimizu J, Ohkoshi N, Kanazawa I, Mizusawa H: Distal myopathy with rimmed vacuoles: novel mutations in the GNE gene. Neurology 2002;59:451-454.

-6 Tomimitsu H, Shimizu J, Ishikawa K, Ohkoshi N, Kanazawa I, Mizusawa H: Distal myopathy with rimmed vacuoles (DMRV): new GNE mutations and splice variant. Neurology 2004;62:1607-1610.

>7 Noguchi S, Keira Y, Murayama K, Ogawa M, Fujita M, Kawahara G, Oya Y, Imazawa M, Goto Y, Hayashi YK, Nonaka I, Nishino I: Reduction of UDP- $N$-acetylglucosamine 2-epimerase/N-acetylmannosamine kinase activity and sialylation in distal myopathy with rimmed vacuoles. J Biol Chem 2004;279:11402-11407.

8 Ro LS, Lee-Chen GJ, Wu YR, Lee M, Hsu PY, Chen CM: Phenotypic variability in a Chinese family with rimmed vacuolar distal myopathy. J Neurol Neurosurg Psychiatry 2005;76:752-755.

-9 Nishino I, Malicdan MC, Murayama K, Nonaka I, Hayashi YK, Noguchi S: Molecular pathomechanism of distal myopathy with rimmed vacuoles. Acta Myol 2005;24:80-83.

10 Liewluck T, Pho-lam T, Limwongse C, Thongnoppakhun W, Boonyapisit K, Raksadawan N, Murayama K, Hayashi YK, Nishino I, Sangruchi T: Mutation analysis of the GNE gene in distal myopathy with rimmed vacuoles (DMRV) patients in Thailand. Muscle Nerve 2006;34:775-778.

11 Chu CC, Kuo HC, Yeh TH, Ro LS, Chen SR, Huang CC: Heterozygous mutations affecting the epimerase domain of the GNE gene causing distal myopathy with rimmed vacuoles in a Taiwanese family. Clin Neurol Neurosurg 2007;109:250-256.

12 Malicdan MC, Noguchi S, Hayashi YK, Nonaka I, Nishino I: Prophylactic treatment with sialic acid metabolites precludes the development of the myopathic phenotype in the DMRV-hIBM mouse model. Nat Med 2009;15:690-695. 